\title{
Effect of methylprednisolone on the activities of caspase-3, $-6,-8$ and -9 in rabbits with acute spinal cord injury
}

\author{
HONG-SHENG LIN ${ }^{1,2}$, ZHI-SHENG JI ${ }^{1,2}$, LI-HENG ZHENG ${ }^{3}$, GUO-QING GUO ${ }^{4}$, \\ BIAO CHEN $^{1,2}, \mathrm{HAO} \mathrm{WU}^{1,2}$ and GUO-WEI ZHANG ${ }^{1,2}$ \\ ${ }^{1}$ Department of Orthopedics, First Affiliated Hospital of Jinan University, Guangzhou 510630; \\ ${ }^{2}$ Institute of Orthopedic Diseases, Jinan University, Guangzhou 510632; ${ }^{3}$ Centro Hospitalar Conde de São Januário, \\ Macao 999078, SAR; ${ }^{4}$ Department of Human Anatomy, Jinan University, Guangzhou 510630, P.R. China
}

Received February 11, 2012; Accepted March 28, 2012

DOI: $10.3892 /$ etm.2012.552

\begin{abstract}
The present study aimed to investigate the effect of methylprednisolone (MP) on the activities of caspase-3, -6, -8 and -9 in rabbits with acute spinal cord injury (ASCI) and to explore the mechanism underlying the antiapoptotic effect of MP on ASCI. Modified Allen's method was employed to establish the ASCI animal model. The animals were randomly divided into a sham (S; $n=12)$, ASCI $(C ; n=36)$ and MP group (T; $\mathrm{n}=36)$. At 8, 24 and $72 \mathrm{~h}$ and 7, 14 and 28 days after ASCI, the animals were sacrificed and the spinal cord was collected. The absorbance (A) was measured with a microplate reader and the activities of caspase-3, $-6,-8$ and -9 were calculated followed by comparisons among the groups. In the $\mathrm{S}$ group, the activities of the four caspases were low. In the $\mathrm{C}$ and $\mathrm{T}$ groups, the caspase activities increased at $8 \mathrm{~h}$ after injury, peaked at $24 \mathrm{~h}$ and remained at a high level 3 days after injury. However, the caspase activities began to decrease at 7 days after injury and were significantly reduced at 14 and 28 days after ASCI. Furthermore, the caspase activities in the T group were markedly lower than those in the $\mathrm{C}$ group at 8 and $24 \mathrm{~h}$ and 3 and 7 days after surgery $(\mathrm{P}<0.05)$, but significant differences were not observed at 14 and 28 days after injury $(\mathrm{P}>0.05)$. In conclusion, MP exerted an antiapoptotic effect via inhibition of the activities of caspase-3, $-6,-8$ and -9 in an animal model of ASCI.
\end{abstract}

\section{Introduction}

Acute spinal cord injury (ASCI) is a type of disability caused by injury of the central nervous system which results in severe pain in patients and has implications for the family and society. The disability is not completely caused by primary

Correspondence to: Dr Hong-Sheng Lin, Department of Orthopedics, First Affiliated Hospital of Jinan University, Guangzhou 510630, P.R. China

E-mail: honeylily1111@sina.com

Key words: acute spinal cord injury, methylprednisolone, caspase, apoptotic factors injury, as secondary injury also plays a significant role. If the primary injury is not treated appropriately and promptly, a secondary injury may result. This secondary injury may be more severe than the primary injury and often results in long-lasting disability. Currently, it is unfeasible to target the primary injury, thus measures should be taken to reduce the incidence of secondary injury following ASCI. Therefore, the key steps in the early treatment of ASCI are to protect the uninjured spinal cord, reduce or prevent secondary injury and promote the re-construction of spinal cord function. The mechanisms underlying secondary injury in ASCI include i) inflammation in which calcium, opioids, excitatory amino acids and other substances mediate injury; ii) free radicals and vascular factors; and iii) cell apoptosis. In recent years, studies have demonstrated that cell apoptosis is crucial in neurological injury following ASCI (1). During apoptosis, members of the caspase family are major participants and caspase activation is a key event in apoptosis.

Methylprednisolone (MP) is the only effective agent used in ASCI treatment, but the therapeutic mechanism of MP remains unclear. A previous study (2) revealed that the therapeutic effectiveness of MP may be attributed to the inhibition of inflammation, attenuation of edema, suppression of vascular activity and prostaglandin activity, increase in blood flow in the spinal cord, inhibition of reactive oxygen species (ROS) and lipid peroxidation, stabilization of cell and lysosomal membranes, reversal of intracellular calcium accumulation, increase in $\mathrm{Na}^{+} \mathrm{K}^{+}$-dependent ATPase activity, increase in resting potential and excitability of spinal motor fibers and promotion of the generation and transduction of nerve impulses in the spinal cord. However, the effect of MP on apoptosis in secondary ASCI and its related mechanisms are poorly understood. The present study aimed to observe apoptosis in the spinal cord of ASCI animals following MP treatment and the activities of apoptosis-related factors (caspase-3, -6, -8 and -9) were also measured in order to investigate the antiapoptotic effect of MP and its mechanism in the treatment of ASCI.

\section{Materials and methods}

ASCI animal model and grouping. Healthy adult New Zealand rabbits weighing 2.0-3.0 kg (males or females) were purchased 
from the Experimental Animal Center (Guangzhou, China). The animals were divided into three groups. In the sham (S) group, the spinal cord was exposed but not injured. In the ASCI group (C), the spinal cord was exposed and the modified Allen's method was employed to cause injury to the spinal cord, but the animals were not treated. In the MP (T) group, ASCI was introduced to animals using the same procedures and the animals were then intravenously (ear vein) treated with $\mathrm{MP}$ at $30 \mathrm{mg} / \mathrm{kg}$ immediately following injury and thereafter at $5.4 \mathrm{mg} /(\mathrm{kg} \cdot \mathrm{h})$ every $2.5 \mathrm{~h}$ within the subsequent $23 \mathrm{~h}$. Following surgery, the animals were housed independently and the bladder was massaged regularly (4-5 times daily until urination recovered). For all animals, intramuscular injection with penicillin (800,000 U) was performed for 3 consecutive days to prevent infection.

Sample collection. At $8 \mathrm{~h}$ and 1, 3, 7, 14 and 28 days after injury, the animals were sacrificed and the lesioned spinal cords were collected. In brief, the animals were weighed, anesthetized, placed in a prone position and fixed followed by skin preparation. An incision was made in the back, the skin and subcutaneous tissues were isolated and the spinal cord was exposed. The lesioned spinal cord $1.5-2.0 \mathrm{~cm}$ in length was collected. The animals were then sacrificed by injection of air through the ear vein. The spinal cord was then added to lysis buffer $(5 \mathrm{mg} / 100 \mu \mathrm{l})$ followed by homogenation on ice. The homogenate was then transferred into a $1.5-\mathrm{ml}$ centrifuge tube followed by lysis on ice for a further $5 \mathrm{~min}$. Centrifugation was performed at $14,000 \mathrm{rpm}$ at $4^{\circ} \mathrm{C}$ for $10-15 \mathrm{~min}$. The supernatant was collected and transferred into a pre-cooled centrifuge tube which was stored at $-70^{\circ} \mathrm{C}$ prior to the detection of caspase activities.

Detection of the activities of caspase-3, -6, -8 and -9 by ELISA. The detection of caspase activities was performed according to the manufacturer's instructions. For example, for the detection of caspase- 3 activity, the procedure was as follows. i) The dilution solution was prepared according to the ratio of $0.9 \mathrm{ml}$ of detection buffer to $0.1 \mathrm{ml}$ of lysis buffer; ii) $p$-nitroaniline $(p \mathrm{NA})(10 \mathrm{mM})$ was diluted to $0,10,20,50,100$ and $200 \mu \mathrm{M}$ with the dilution solution and used as standard samples. iii) A volume $(100 \mu \mathrm{l})$ of each standard sample was collected and the absorbance was measured at $405 \mathrm{~nm}$ (A405). The actual A405 of $p$ NA, based on which the $p$ NA standard curve was delineated, was acquired by subtracting the A405 of the blank control (without $p$ NA) from that of each standard sample. iv) The $p$ NA was mixed in Ac-DEVD- $p$ NA $(2 \mathrm{mM})$ of appropriate volume and the mixture was kept on ice until use. v) The mixture was incubated at $37^{\circ} \mathrm{C}$ for $1-2 \mathrm{~h}$. When the color changed, A405 was measured. When the color remained unchanged, the incubation was prolonged. vi) The absorbance was measured at $405 \mathrm{~nm}$. The A 405 of $p$ NA generated by caspase- 3 was calculated by subtracting the A405 of the blank control from that of the samples. The content of $p$ NA was determined according to the standard curve on the basis of the A405 of $p$ NA. vii) The definition of caspase-3 activity (Chemicon, Temecula, CA, USA) was that when the substrate was saturated; one unit of enzyme activity is the amount of caspase- 3 that catalyzes $1 \mathrm{nmol}$ of Ac-DEVD- $p \mathrm{NA}$ into $1 \mathrm{nmol}$ of $p \mathrm{NA}$ at $37^{\circ} \mathrm{C}$. The caspase- 3 activity of each sample was calculated.
Statistical analysis. Statistical analysis was performed using SPSS version 16.0 statistical software (SPSS, Inc., Chicago, IL, USA). Comparisons between the $\mathrm{T}$ and $\mathrm{C}$ groups were performed using a t-test, and $\mathrm{P}<0.05$ was considered to indicate a statistically significant result.

\section{Results}

In the $\mathrm{S}$ group, the activities of caspase- $3,-6$ and -8 were $3.127,0.029$ and $2.733 \times 10^{4} \mathrm{U}$, respectively, at $8 \mathrm{~h}$ after surgery; $3.173,0.029$ and $2.777 \times 10^{4} \mathrm{U}$, respectively, at $24 \mathrm{~h}$ after surgery; $3.14,0.028$ and $2.787 \times 10^{4} \mathrm{U}$ at 3 days after surgery, respectively; $3.143,0.028$ and $2.777 \times 10^{4} \mathrm{U}$, respectively, at 7 days after surgery; 3.16, 0.029 and $2.747 \times 10^{4} \mathrm{U}$, respectively, at 14 days after surgery; 3.13, 0.287 and $2.787 \times 10^{4} \mathrm{U}$, respectively, at 28 days after surgery. The caspase- 9 activity was extremely low at $24 \mathrm{~h}$ after surgery and was undetectable at the remaining time points. In the $\mathrm{C}$ group, the activities of caspase- $3,-6,-8$ and -9 were markedly increased at $8 \mathrm{~h}$ after surgery and peaked at $24 \mathrm{~h}$. At 3 days after injury, the activities of the caspases remained high, but were lower than those at $24 \mathrm{~h}$. At 7 days after injury, the activities were markedly reduced and remained at a low level at 14 and 28 days after ASCI. In the T group, the activities of caspase- $3,-6,-8$ and -9 were significantly increased at $8 \mathrm{~h}$ after surgery and reached a maximal level at $24 \mathrm{~h}$. At 3 days after surgery, the activities remained high but were lower than those at $24 \mathrm{~h}$ after surgery and those in the $\mathrm{C}$ group. At 7 days after surgery, the activities were markedly reduced and remained at a low level at 14 and 28 days after surgery. The activities of caspase- $3,-6,-8$ and -9 are shown in Tables I-IV. When compared with the C group, the caspase activities were markedly lowered in the $\mathrm{T}$ group $(\mathrm{P}<0.05)$. However, no marked difference in caspase activities was noted between the two groups at 14 and 28 days after surgery ( $\mathrm{P}>0.05$; Tables I-IV and Fig. 1).

\section{Discussion}

Apoptosis may occur in a death receptor-, mitochondria- or endoplasmic reticulum-dependent manner. Caspase activation has been regarded as a key process in apoptosis. To date, a total of 14 caspases have been identified, termed caspases 1-14. On the basis of the role they play in apoptosis, caspases are divided into initiator and effector caspases. The initiator caspases include caspase-2, - $8,-9$ and -10 , which are located upstream of the apoptosis cascade reaction. These caspases have long N-terminals, initiate apoptosis and caspase activation and regulate downstream caspases. Effector caspases are located downstream of the apoptosis cascade reaction and include caspase- $3,-6$ and -7. Following the activation of caspase- $3,-6$ and -7 by upstream initiators, these caspases act on substrates resulting in the changes in cellular biochemistry and morphology which are characteristic of apoptosis. Other caspases, including caspase-1, $-4,-5,-13$ and -14 are mainly involved in inflammation and exert an adjunctive effect on apoptosis. Apoptosis occurs in different ways as a result of the interaction of multiple factors $(3,4)$. In the present study, we measured the activities of initiator caspases (caspase- 8 and -9) and effector caspases (caspase-3 and -6) aiming to investigate the antiapoptotic effect of MP in the treatment of ASCI. 
Table I. Caspase-3 activity in T group and $\mathrm{C}$ group.

\begin{tabular}{lcrcc}
\hline Time point & Group & Activity $(\overline{\mathrm{x}} \pm \mathrm{SD})$ & $\mathrm{t}$ & P-value \\
\hline $8 \mathrm{~h}$ & $\mathrm{C}$ & $17.0789 \pm 0.86519$ & 2.705 & 0.016 \\
& $\mathrm{~T}$ & $16.2244 \pm 0.38636$ & & \\
$24 \mathrm{~h}$ & $\mathrm{C}$ & $35.2322 \pm 0.33559$ & 22.908 & 0.000 \\
& $\mathrm{~T}$ & $29.4311 \pm 0.68157$ & & \\
3 days & $\mathrm{C}$ & $21.5644 \pm 0.86794$ & 14.126 & 0.000 \\
& $\mathrm{~T}$ & $16.8678 \pm 0.49157$ & & \\
7 days & $\mathrm{C}$ & $13.3011 \pm 0.27310$ & 23.532 & 0.000 \\
& $\mathrm{~T}$ & $11.0100 \pm 0.10356$ & & \\
14 days & $\mathrm{C}$ & $4.8633 \pm 0.17407$ & 1.834 & 0.085 \\
& $\mathrm{~T}$ & $4.7133 \pm 0.17292$ & & \\
28 days & $\mathrm{C}$ & $3.1856 \pm 0.04246$ & 0.553 & 0.588 \\
& $\mathrm{~T}$ & $3.1744 \pm 0.04275$ & & \\
\hline
\end{tabular}

t, t-value; T, treatment; $\mathrm{C}$, control.

Table II. Caspase-6 activity in T group and C group.

\begin{tabular}{lcccc}
\hline Time point & Group & Activity $(\overline{\mathrm{x}} \pm \mathrm{SD})$ & $\mathrm{t}$ & P-value \\
\hline $8 \mathrm{~h}$ & $\mathrm{C}$ & $0.059778 \pm 0.0031929$ & 2.743 & 0.014 \\
& $\mathrm{~T}$ & $0.056333 \pm 0.020000$ & & \\
$24 \mathrm{~h}$ & $\mathrm{C}$ & $0.20944 \pm 0.0068759$ & 9.834 & 0.000 \\
& $\mathrm{~T}$ & $0.180778 \pm 0.0054032$ & & \\
3 days & $\mathrm{C}$ & $0.105667 \pm 0.0031225$ & 22.984 & 0.000 \\
& $\mathrm{~T}$ & $0.069778 \pm 0.0034921$ & & \\
7 days & $\mathrm{C}$ & $0.056000 \pm 0.0026926$ & 12.544 & 0.000 \\
& $\mathrm{~T}$ & $0.041667 \pm 0.0021213$ & & \\
14 days & $\mathrm{C}$ & $0.034889 \pm 0.0010541$ & 2.012 & 0.061 \\
& $\mathrm{~T}$ & $0.033889 \pm 0.0010541$ & & \\
28 days & $\mathrm{C}$ & $0.027333 \pm 0.002000$ & 0.134 & 0.895 \\
& $\mathrm{~T}$ & $0.027222 \pm 0.0014814$ & & \\
\hline
\end{tabular}

t, t-value; T, treatment; $\mathrm{C}$, control.

Caspase-3 is a critical participant in apoptosis. In mammals, caspase- 3 corresponds to CED-3 in nematodes (5). Caspase- 3 is an important effector caspase and is the final executive caspase in various apoptosis pathways (6). The activated caspase-3 is able to digest or cleave proteins and kinases which are involved in cell structure, cell cycle and DNA replication, resulting in the activation or inactivation of these kinases and proteins and subsequent apoptosis. Sîrbulescu and Zupanc applied 1,3-cyclohexanedione to inhibit caspase activation and the results revealed that the number of apoptotic cells at $24 \mathrm{~h}$ and 5 and 30 days after surgery was markedly lower than that in the control group (7). Caspase- 3 is activated by endogenous and exogenous pathways. In the exogenous pathway, the death receptor carrying death signals binds to the corresponding death ligand in the cell membrane, resulting in its oligomerization. This then recruits Fas-related protein and caspase- 8 to
Table III. Caspase- 8 activity in $\mathrm{T}$ group and $\mathrm{C}$ group.

\begin{tabular}{lcrcc}
\hline Time point & Group & Activity $(\overline{\mathrm{x}} \pm \mathrm{SD})$ & $\mathrm{t}$ & P-value \\
\hline $8 \mathrm{~h}$ & $\mathrm{C}$ & $16.2733 \pm 0.22533$ & 2.648 & 0.024 \\
& $\mathrm{~T}$ & $16.0600 \pm 0.08746$ & & \\
$24 \mathrm{~h}$ & $\mathrm{C}$ & $25.7744 \pm 0.12943$ & 45.486 & 0.000 \\
& $\mathrm{~T}$ & $19.5878 \pm 0.38697$ & & \\
3 days & $\mathrm{C}$ & $18.0500 \pm 0.08292$ & 54.222 & 0.000 \\
& $\mathrm{~T}$ & $16.4133 \pm 0.03640$ & & \\
7 days & $\mathrm{C}$ & $11.1156 \pm 0.10466$ & 69.433 & 0.000 \\
& $\mathrm{~T}$ & $8.0167 \pm 0.08352$ & & \\
14 days & $\mathrm{C}$ & $2.9478 \pm 0.03701$ & 0.966 & 0.349 \\
& $\mathrm{~T}$ & $2.9311 \pm 0.03621$ & & \\
28 days & $\mathrm{C}$ & $2.7933 \pm 0.03279$ & 1.710 & 0.107 \\
& $\mathrm{~T}$ & $2.7700 \pm 0.02449$ & & \\
\hline
\end{tabular}

t, t-value; T, treatment; C, control.

Table IV. Caspase-9 activity in T group and C group.

\begin{tabular}{lrrrr}
\hline Time point & Group & Activity $(\overline{\mathrm{x}} \pm \mathrm{SD})$ & $\mathrm{t}$ & P-value \\
\hline $8 \mathrm{~h}$ & $\mathrm{C}$ & $6.6189 \pm 0.05776$ & 2.243 & 0.039 \\
& $\mathrm{~T}$ & $6.5689 \pm 0.03371$ & & \\
$24 \mathrm{~h}$ & $\mathrm{C}$ & $11.2422 \pm 0.15123$ & 15.017 & 0.000 \\
& $\mathrm{~T}$ & $9.5289 \pm 0.30706$ & & \\
3 days & $\mathrm{C}$ & $8.7822 \pm 0.23679$ & 18.515 & 0.000 \\
& $\mathrm{~T}$ & $6.9700 \pm 0.17364$ & & \\
7 days & $\mathrm{C}$ & $5.8211 \pm 0.47635$ & 7.401 & 0.000 \\
& $\mathrm{~T}$ & $3.8078 \pm 0.66272$ & & \\
14 days & $\mathrm{C}$ & $1.8178 \pm 0.10837$ & 0.931 & 0.366 \\
& $\mathrm{~T}$ & $1.7533 \pm 0.17720$ & & \\
28 days & $\mathrm{C}$ & $0.0011 \pm 0.00333$ & 1 & 0.332 \\
& $\mathrm{~T}$ & $0.0000 \pm 0.00000$ & & \\
\hline
\end{tabular}

t, t-value; T, treatment; C, control.

form the death-inducing signaling complex. Caspase- 8 then self-activates and the activated caspase- 8 directly activates caspase-3 (8). Caspase-3 is also activated by cytochrome $\mathrm{C}$ (CytC) leaked from mitochondria, which is known as the endogenous pathway. The activation of caspase-3 not only leads to apoptosis but acts on pro-caspase- 8 resulting in caspase- 8 activation, which then forms a positive feedback loop and leads to a cascade reaction (9). In the present study, our results revealed that at 8 and $24 \mathrm{~h}$ and 3 and 7 days after the MP treatment of ASCI, caspase- 3 activity was markedly reduced. That is, the activity of the executive caspases was inhibited, which then alleviated the cleavage of other proteins and kinases by caspase- 3 and reduced the activation or inactivation of these proteins and kinases.

Caspase- 6 was first identified and cloned by FernandesAlnemri et al (10). Caspase- 6 is encoded by the gene located at 
A

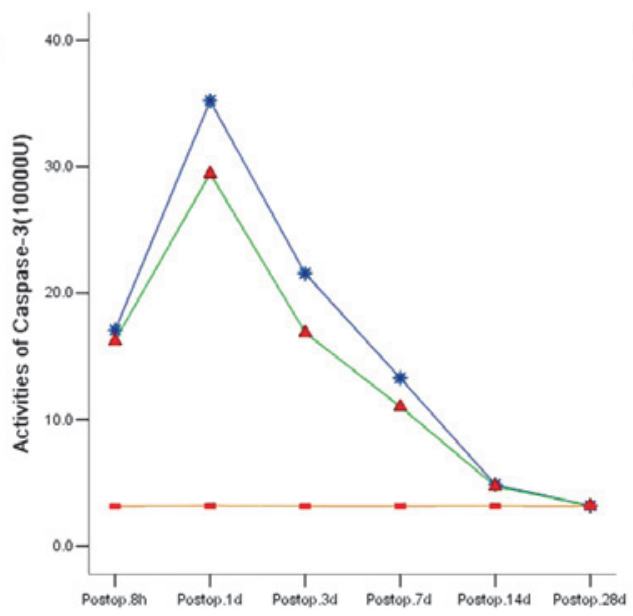

C

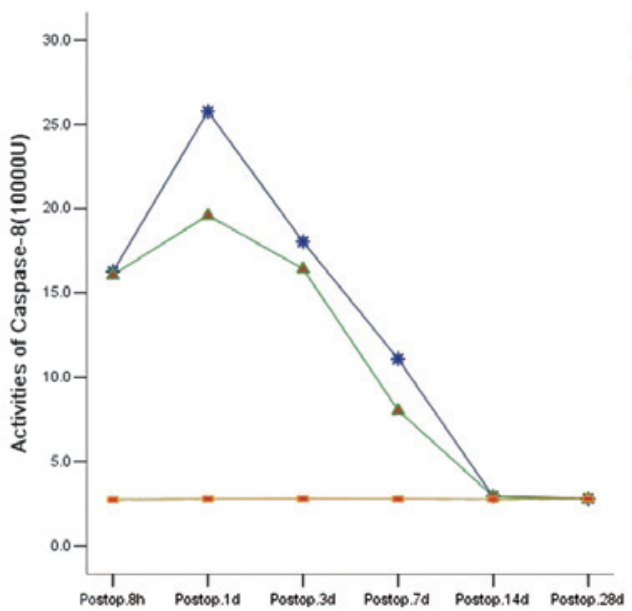

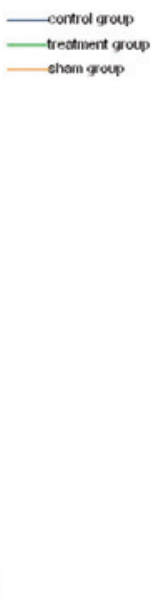

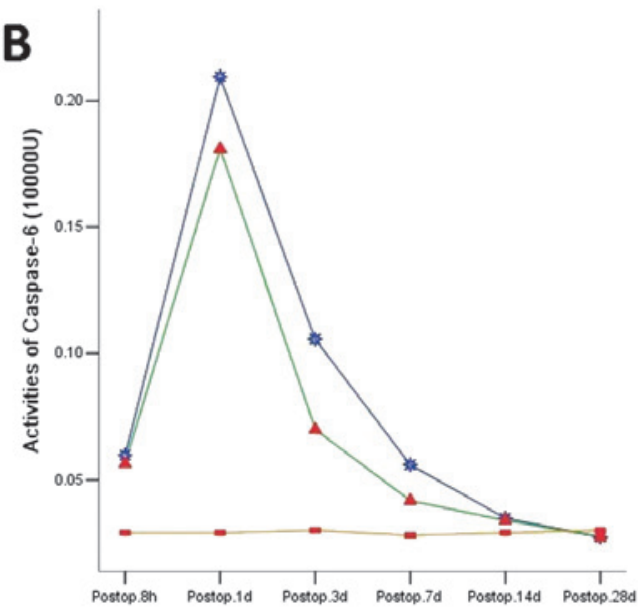

D

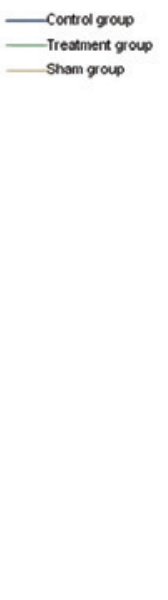

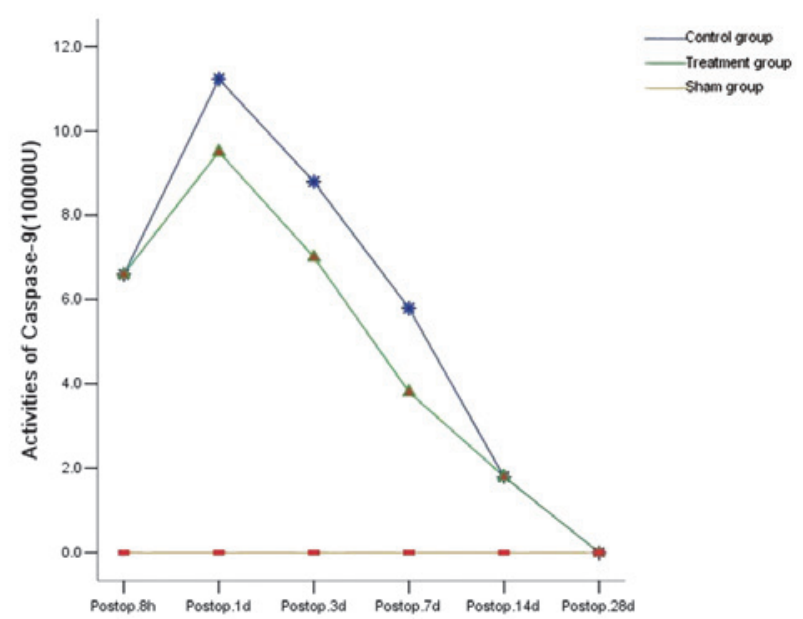

Figure 1. Caspase-3, -6, -8 and -9 activities in different groups. (A) Caspase-3; (B) caspase-6; (C) caspase-8; (D) caspase-9. The caspase-3, -6, -8 and -9 activity increased significantly at $8 \mathrm{~h}$ after surgery, but the increase in the $\mathrm{T}$ group was markedly lower than that in the $\mathrm{C}$ group (P<0.05). At $24 \mathrm{~h}$ and 3 and 7 days, the caspase- $3,-6,-8$ and -9 activity in the T group was markedly lower than that in the $\mathrm{C}$ group $(\mathrm{P}<0.01)$. At 14 and 28 days after injury, the caspase-3, $-6,-8$ and -9 activity was markedly reduced when compared with that at other time points but no significant difference was found between the $\mathrm{C}$ and $\mathrm{T}$ groups $(\mathrm{P}>0.05)$. At 28 days after injury, the caspase- 9 activity was undetectable and comparable to that in the blank control group. T, treatment; C, control.

4q25 and has a molecular weight of $34 \mathrm{kDa}$. Caspase- 6 mainly exists in the form of zymogen in the cytoplasm and a fraction of caspase- 6 is located in the nucleus. In apoptosis, caspase- 6 is located downstream of the caspase cascade reaction, as is caspase-3. Caspase- 6 cleaves lamin A, leading to chromosome condensation and resulting in apoptosis. Although caspase- 6 and -3 are located downstream of the apoptosis cascade pathway, the interaction between caspase- 6 and -3 remains unclear (11). There is evidence which indicates that the activation of caspase- 3 is earlier than that of caspase- 6 and is closely correlated with the cleavage of procaspase- 6 into caspase- 6 . In addition, activated caspase- 6 may in turn activate caspase-3, forming a positive feedback loop in the caspase-3 activity. Caspase- 6 and -3 are effector caspases and are located downstream of the apoptosis cascade reaction, but they are not in the same reaction chain. The two proteins function independently but are correlated with each other. In the absence of caspase- 6 activation, caspase- 3 activation alone may also induce apoptosis. Furthermore, caspase- 6 activation alone is also sufficient to induce apoptosis in the absence of caspase- 3 activation. However, apoptosis in cells usually occurs via more than one pathway. In the present study, our results showed that the activities of caspase- 6 and -3 were inhibited following MP treatment, which suggests that MP suppresses more than one apoptosis-related pathway, exerting an antiapoptotic effect. The inhibition of one pathway may also block the interaction of this pathway with other pathways. That is, the interaction between caspase- 6 and -3 is inhibited, which also indirectly attenuates apoptosis.

Caspase- 8 is regarded as a significant member of the caspase family that induces apoptosis in mammals (12). Following activation, caspase- 8 cleaves and activates caspase- 3 downstream of the caspase cascade reaction. Caspase- 3 serves as a substrate of caspase- 8 and activated caspase- 3 then acts on certain proteinases in the cell, resulting in their activation or inactivation. This may cause damage to lamin and lead to apoptosis $(13,14)$. In addition, caspase-3 in turn activates pro-caspase- 8 , forming a positive feedback loop which leads to the caspase cascade reaction. In the present study, at 8 and $24 \mathrm{~h}$ and 3 and 7 days after MP treatment of ASCI, the caspase- 3 
and -8 activities were markedly decreased and, furthermore, the interaction between caspase- 8 and -3 was also attenuated, which compromised the activation of caspase- 3 by caspase- 8 and the positive feedback loop between caspase-3 and -8 . This inhibited the caspase cascade reaction and resulted in the attenuation of apoptosis. The activated caspase- 8 also cleaves the Bid, which is a member of the Bcl-2 family. The cleaved tBid exposes the $\mathrm{C}$ terminal which then binds to the mitochondria. This transmits the apoptosis signals from the cytoplasm to the mitochondria, resulting in the release of CytC from the mitochondria (15), which is a key step in the endogenous apoptosis pathway. The results of previous studies have confirmed that CytC expression is positively correlated with apoptosis. There is evidence which suggests that fluorouracil promotes the release of $\mathrm{CytC}$ from mitochondria to induce the apoptosis of liver cancer cells (16). The CytC released from mitochondria is able to form complexes with caspase- 1 and -9 , which may activate caspase-3, -6 and -7 in the presence of dATP and ATP and induce apoptosis. The present study found that the caspase- 8 activity was markedly inhibited following the MP treatment of ASCI, which may attenuate the interaction between members of the Bcl-2 family and mitochondria, reduce the transmission of apoptosis signals into the mitochondria and finally compromise mitochondria-dependent apoptosis. MP inhibits the activity of caspase-8, which not only suppresses the caspase cascade reaction but indirectly inhibits mitochondria-dependent apoptosis.

Caspase- 9 is a key proteinase in the mitochondria-dependent pathway and a significant initiator caspase in the caspase cascade reaction. When tissues are injured, cells undergo ischemia and hypoxia, which may increase mitochondrial permeability. The proteins in the mitochondrial inner and outer membranes (including $\mathrm{CytC}$ ) can then be released into the cytoplasm (16). CytC in the cytoplasm forms a poly-complex with Apaf-1 which then activates pro-caspase-9. The activated caspase- 9 may further activate caspase-3, initiating the caspase cascade reaction. Caspase- 3 then cleaves substrates, resulting in apoptosis. A previous study has demonstrated that activated caspase-3 acts on pro-caspase-9, resulting in caspase- 9 activation and forming a feedback loop which may lead to the caspase cascade (18). Colak et al (19) applied z-LEHD-fmk, a caspase- 9 inhibitor, in the treatment of spinal cord injury in rats. Electron microscopy confirmed that z-LEHD-fmk was able to protect neurons, glial cells, myelin, axons and intracellular organelles. In the present study, the results showed that caspase-9 activity was markedly reduced following MP treatment of ASCI, which may inhibit the activation of downstream caspases and attenuate apoptosis. On the other hand, MP also inhibited caspase-3 activity, which may suppress the interaction between caspase- 3 and the other caspases. All these effects finally result in the attenuation of apoptosis in ASCI following MP treatment.

In the present study, we applied MP in the treatment of ASCI and the activities of caspase- $3,-6,-8$ and -9 were measured at different time points following the injury. The results showed that at 8 and $24 \mathrm{~h}$ and 3 and 7 days after injury, the activities of all caspases were markedly increased but the increase in the MP treatment group was lower than that in the untreated animals $(\mathrm{P}<0.05)$. At 14 days after injury, the activities of all caspases were markedly reduced when compared with those at the former four time points, but a marked difference was not noted between the MP treatment and the untreated groups $(\mathrm{P}>0.05)$. At 28 days after injury, the activities of all the caspases were comparable to those in the blank control group or were undetectable. These findings demonstrate that MP is able to inhibit the activity of these caspases and suppress their interaction, which indirectly inhibits the mitochondria-dependent apoptosis pathway and the caspase cascade reaction in apoptosis, resulting in an antiapoptotic effect. However, MP was not capable of delaying the activation or causing the inactivation of these caspases.

Thus, we speculate that MP reduces the activities of certain significant molecules in the caspase cascade reaction (including caspase-3, -6, -8 and -9) and compromises the interaction between these molecules, which leads to the inhibition of the apoptosis cascade and indirectly inhibits the mitochondria-dependent apoptosis pathway, resulting in an antiapoptotic effect. Finally, secondary spinal cord injury is attenuated. However, MP cannot completely inactivate or delay the activation of these caspases.

\section{Acknowledgements}

This study was supported by the Science and Technology Project of Guangdong Province (2010/170,2010B03160010) and Science and Technology Development Fund of Macao (026/2010/A).

\section{References}

1. Lou J, Lenke LG, Ludwig FJ and O'Brien MF: Apoptosis as a mechanism of neuronal cell death following acute experimental spinal cord injury. Spinal Cord 6: 683-690, 1998

2. Torres S, Salgado-Ceballos H, Torres JL, et al: Early metabolic reactivation versus antioxidant therapy after a traumatic spinal cord injury in adult rats. Neuropathology 30: 36-43, 2010

3. Nakagawa T, Zhu H, Morishima N, Li E, Xu J, Yankner BA and Yuan J: Caspase-12 mediates endoplasmic-reticulum-specific apoptosis and cytotoxicity by amyloid-beta. Nature 403: 98-103, 2000.

4. Srinivasula SM, Hegde R, Saleh A, et al: A conserved XIAPinteraction motif in caspase-9 and Smac/DIABLO regulates caspase activity and apoptosis. Nature 409: 112-116, 2001.

5. Breckenridge DG, Kang BH, Kokel D, Mitani S, Staehelin LA and Xue D: Caenorhabditis elegans drp-1 and fis-2 regulate distinct cell-death execution pathways downstream of ced-3 and independent of ced-9. Mol Cell 31: 586-597, 2008.

6. Faubel S and Edelstein CL: Caspases as drug targets in ischemic organ injury. Curr Drug Targets Immune Endocr Metabol Disord 5: 269-287, 2005.

7. Sîrbulescu RF and Zupanc GK: Inhibition of caspase-3-mediated apoptosis improves spinal cord repair in a regeneration-competent vertebrate system. Neuroscience 171: 599-612, 2010.

8. Lahiry L, Saha B, Chakraborty J, et al: The aflavins target Fas/caspase- 8 and Akt/pBad pathways to induce apoptosis in p53-mutated human breast cancer cells. Carcinogenesis 31: 259-268, 2010.

9. Micheau O, Thome M, Schneider P, et al: The long form of FLIP is an activator of caspase- 8 at the Fas death-inducing signaling complex. J Biol Chem 277: 45162-45171, 2002.

10. Fernandes-Alnemri T, Litwack G and Alnemri ES: Mch2, a new member of the apoptotic Ced-3/Ice cysteine protease gene family. Cancer Res 55: 2737-2742, 1995.

11. Warby SC, Doty CN, Graham RK, et al: Activated caspase-6 and caspase-6-cleaved fragments of huntingtin specifically colocalize in the nucleus. Hum Mol Genet 17: 2390-2404, 2008.

12. Cheng G, Wei L, Zhi-Dan S, Shi-Guang Z and Xiang-Zhen L: Atorvastatin ameliorates cerebral vasospasm and early brain injury after subarachnoid hemorrhage and inhibits caspasedependent apoptosis pathway. BMC Neurosci 21: 7, 2009. 
13. Li YH, Wang C, Meng K, Chen LB and Zhou XJ: Influence of survivin and caspase-3 on cell apoptosis and prognosis in gastric carcinoma. World J Gastroenterol 10: 1984-1988, 2004.

14. Mandal D, Mazumder A, Das P, Kundu M and Basu J: Fas-, caspase 8-, and caspase 3-dependent signaling regulates the activity of the aminophospholipid Translocase and phosphatidylserine externalization in human erythrocytes. J Biol Chem 280: 39460-39467, 2005

15. Miller CP, Ban K, Dujka ME, McConkey DJ, Munsell M, Palladino $\mathrm{M}$ and Chandra J: NPI-0052, a novel proteasome inhibitor, induces caspase-8 and ROS-dependent apoptosis alone and in combination with HDAC inhibitors in leukemia cells. Blood 110: 267-277, 2007.

16. Yang JQ: The role of cytochrome $\mathrm{C}$ in the apoptosis of hepatoma cell induced by fluorouracil. Zhougguo Pu Tong Waike Zazhi 6: 1081-1084, 2007 (In Chinese).
17. Wu KL, Hsu C and Chan JY: Impairment of the mitochondrial respiratory enzyme activity triggers sequential activation of apoptosis-inducing factor-dependent and caspase-dependent signaling pathways to induce apoptosis after spinal cord injury. J Neurochem 101: 1552-1566, 2007.

18. Fujita E, Egashira J, Urase K, Kuida K and Momoi T: Caspase-9 processing by caspase-3 via a feed-back amplification loop in vivo. Cell Death Differ 8: 335-344, 2001.

19. Colak A, Karaoğlan A, Barut S, Köktürk S, Akyildiz AI and Taşyürekli M: Neuroprotection and functional recovery after application of the caspase-9 inhibitor z-LEHD-fmk in a rat model of traumatic spinal cord injury. J Neurosurg Spine 2: 327-334, 2005. 